\title{
ULTRAESTRUCTURA DE ESPOROFITOS JOVENES DE Macrocystis pyrifera (Linnaeus) C. Agardh (PHAEOPHYCEAE, LAMINARIALES).
}

\author{
César A. Córdova ${ }^{1}$, Nélida S. Tejada ${ }^{1 \dagger}$ y Mariela A. González ${ }^{2}$
}

\section{RESUMEN}

Se investigó el desarrollo anatómico de esporofitos jóvenes de Macrocystis pyrifera obtenidos a partir de cigotos en cultivos de laboratorio. Los talos eran monostromáticos luego de dos semanas de crecimiento y las células eran similares entre sí, fotosintéticas y conectadas por campos de puntuación primarios. A las cinco semanas, los talos eran poliestromáticos y estaban diferenciados internamente en meristodermo, corteza y médula. Filamentos cribosos longitudinales aparecieron en la médula, desarrollándose de la corteza interna y rodeados por una matriz intercelular. Cada elemento criboso presentaba un núcleo, cloroplastos y varios plasmodesmos, los cuales estaban distribuidos más o menos at azar en paredes transversales o agrupados en campos de puntuación primarios en paredes longitudinates. Luego de nueve semanas, los talos eran más gruesos y estaban diferenciados morfológicamente en una lámina, estípite y grampón rizoidal. Además de los filamentos cribosos, aparecieron filamentos menores o "hifas" en la médula. Se discute el desarrollo del esporofito joven y la relación de nuestras observaciones con estudios en otras algas pardas.

\begin{abstract}
The anatomical development of young sporophytes of Macrocystis pyrifera obtained from zygotes in laboratory cultures was investigated. Thalli were monostromatic after two weeks of growth, and ceils were similar, photosynthetic, and connected by primary pit fields.

Thalli were polystromatic and differentiated internally into meristoderm, cortex and medulla in the fifth week. Longitudinal sieve filaments appeared in the medulla developing from the inner cortex and they were surrounded by an intercellular matrix. Individual sieve elements contained a nucleus, chloroplasts and several plasmodesmata, which were more or less randomly distributed in transverse walls or they were grouped in primary pit fields in longitudinal walls. Thalli were thicker and differentiated morphologically into a single lamina, stipe and rhizoidal holdfast after nine weeks. In addition to sieve filaments, smaller filaments or "hyphae" appeared in the medulla. Possible developmental pathways to adult stages and the relationship of our finding with other studies for brown algae are discussed.
\end{abstract}

Key words: alga, Phaeophyceae, Laminariales, Macrocystis, ultraestructura, plasmodesmo, desarrollo.

\section{INTRODUCCION}

La especie Macrocystis pyrifera (L.) C. Ag. (Phaeophyceae, Laminariales, Lessoniaceae) es el alga de mayor tamaño conocida. Habita principalmente en el submareal rocoso y está ampliamente distribuida en las costas subantárticas del hemisferio Sur y en las del Oeste de Norteamérica (Ricker, 1987). En Sudamérica, donde se la conoce comúnmente como «huiro» o «cachiyuyo», habita las costas del Perú, Chile y Argentina (Kühnemann,
1970; 1972; Ramírez \& Santelices, 1991).

El esporofito adulto se caracteriza por exhibir un alto grado de especialización morfoanatómica (Parker, 1971; Lobban, 1978; Schmitz, 1981). Presenta un talo diferenciado en frondas (incluyendo estípite, flotador, lámina) y grampón (Fritsch, 1945; Parker, 1971; Schmitz, 1981) y, desde el punto de vista anatómico, muestra un meristodermo, una corteza de células parenquimáticas y una médula formada por "hifas", tubos cribosos y filamentos trompera, estos últimos asociados a la

\footnotetext{
'Departamento de Botánica, Facultad de Ciencias Biológicas. Universidad Nacional Muyor de San Marcos, 14 0002, Lima, Perú

2Departamento de Boránica, Facultad de Ciencias Valuralcs y Oceanográticas. LIniversidad de Concepción. Casilia 2407, Concepción. Chile
} 
translocación de fotosintatos (Oliver, 1887; Sykes, 1908; Parker \& Diboll, 1966; Parker, 1971; Barrales al., 1981 ; Grenville et al., 1982; Núñez \& Alveal, 1988). Sykes (1908) realizó un decallado estudio del crecimiento y la dilerenciación de talos adulcos de $M$. pyrifera basándose en observaciones sobre plantas macroscópicas. Hasta el momento, no existe una descripción anatómica de estadíos juveniles que permita entender el desarrol Jo de esta especie. Por ello, el objetivo del presence citudio es proporcionar información sobre liu ultraestructura de esporofitos jóvenes de hasta nueve semanas de incubación, obtenidos a partir de cultivos en el laboratorio.

\section{MATERIAL Y METODOS}

Se recolectó esporofitos fértiles de $M$. pyrifera manualmente, mediante buceo SCUBA, del submareal rocoso $(2 \mathrm{~m}$ de profundidad) de Punta de Parra ( $36^{\circ} 40^{\prime} \mathrm{S} ; 72^{\circ}$ $58^{\prime}$ W), Octava Región, Chile, en marzo de 1991. El material, sumergido en agua de mar, fue trasladado al laboratorio en una cámara de aislamiento térmico.

Fragmentos de talo de $2 \times 2 \mathrm{~mm}$ conteniendo soros fueron colocados en placas perri con agua de mar enriquecida con solución de Provasoli y se incubó en una cámara de cultivo a $15^{\circ} \mathrm{C}$, con un foloperíodo de $16: 8(\mathrm{~L}: \mathrm{O})$ y una densidad de flujo forónico de $3.5 \mu \mathrm{E} \mathrm{m}$ $2 \mathrm{~s}^{-1}$. Lueoo de 24 horas y una vez liberadas las meiosporas, se eliminaron los trozos de talo. Los gametofitos resultantes permanecieron en las mismas condiciones de cultivo hasta la aparición de los primeros esporofitos. Los esporofitos fueron colectados con una pipeta Pasteur y se distribuyeron, agrupados de a cuatro, en una placa multicelda, donde se desarrollaron bajo las mismas condiciones anteriores, excepto la densidad de flujo fotónico. que fue de $35, \mathrm{E} \mathrm{m}^{-2} \mathrm{~s}^{-1}$.

Para el estudio al microscopio electrónico de transmisión (MET) se fijaron esporofitos de 1 a 9 semanas de incubación, en una solución de acroleína 10\% y glutaraldehído $10 \%$ en tampón cacodilato de sodio 0,2 M (pH 7.2) durante 12 h. La postfijación se realizó en tetróxido de osmio al $2 \%$ por $2 \mathrm{~h}$. La deshidratación se llevó a cabo con una serie ascendente de acetona, para luego infiltrar el material en resina Spurr por $12 \mathrm{~h}$ a centrifugación lenta (Glauert, 1974). Secfectuaron cortes de 40 a $50 \mathrm{~nm}$ de espesor con cuchilla de vidrio en ultramicrótomo Sorval MT 5000 y se iñeron con acetato de uranilo y citrato de plomo (Reynolds, 1963). Finalmente, fueron observados en un MET Philips EM 200. La medición del diámetro de los poros en las paredes celulares se hicieron en fotografías de más de 1000 aumentos. Cortes de $1 \mu$ m de espesor se colorearon con azul de toluidina al $1 \%$ y se observaron al microscopio óptico.

\section{RESULTADOS}

Los esporofitos de 2 semanas de edad presentan un talo aplanado monostromático (Fig. 1). Las células que to componen tienen una pared delgada, formada principalmente por microfibrillas paralelas a la superficie celular, y sobre su cara externa se deposita una "cutícula» microfibrilar. El núcleo ocupa gran parte del volumen celular, los cloroplastos, de ubicación parietal, son elipsoidalús con 6 a 10 lamelas y se observan una o más vacuolas (Figs. 1 y 2). Entre células contiguas existen campos de puntuación primarios con plasmodesmos de un diámetro promedio de poro de 38,8 nm (Fig. 3).

A las 3 semanas de edad, los talos tienen de 3 a 4 capas celulares de espesor, las que no presentan diferencias molfológicas importantes entre sí (ig. 4). Además, sus características citológicas son similares a las de células del estadío anterior. Los campos de puntuación primarios siguen siendo comúnmente obscrvados (Figs. 5 y 6), con un diámetro promedio de poro de $34,9 \mathrm{~nm}$.

En talos de 5 semanas de edad, ya se observa diferenciación celular. Es posible reconocer células del meristodermo, de la corteza y de la médula (Fig. 7), a la vez que las 

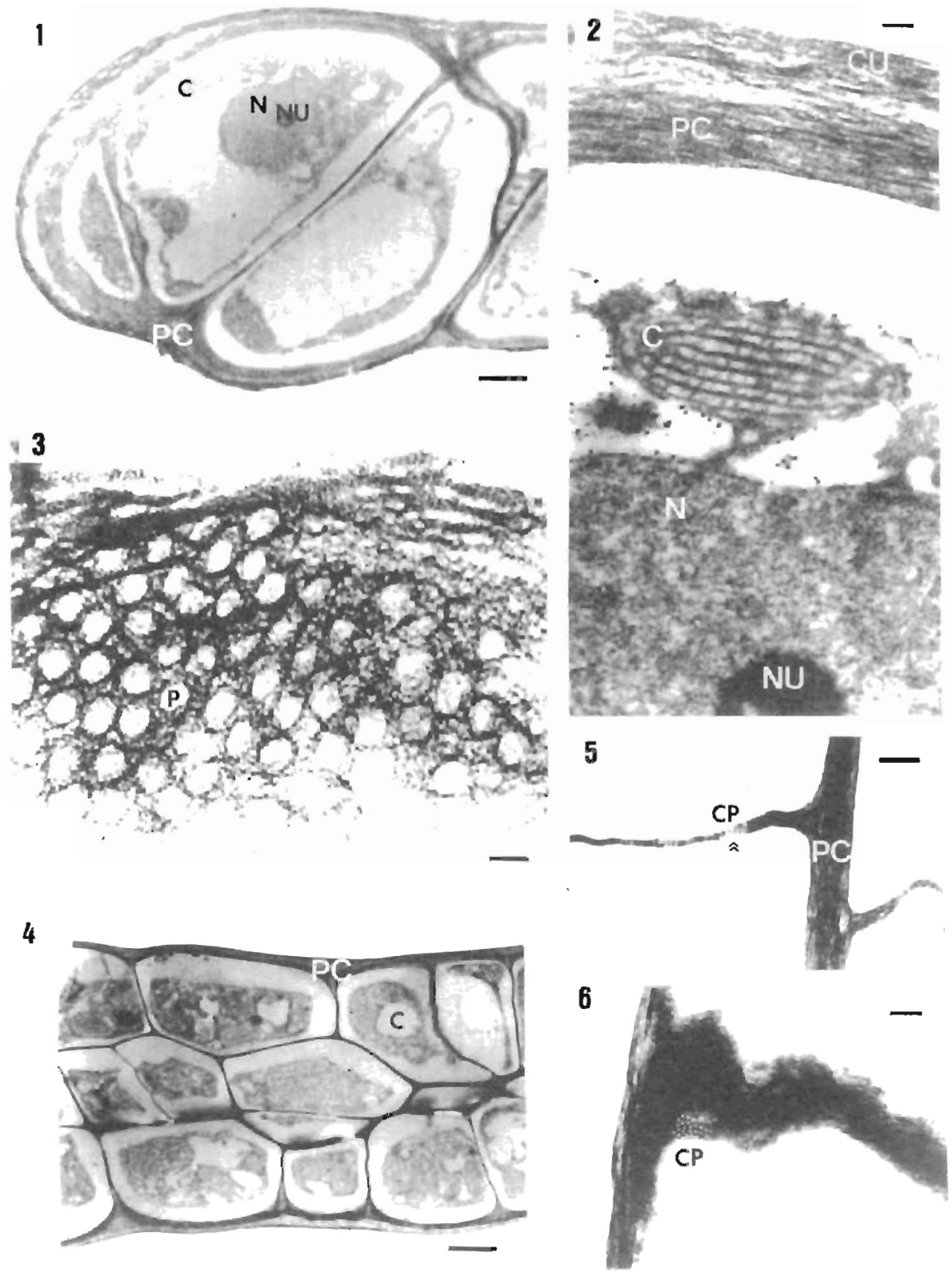

Meristodermo (M), corteza (Co), médula (ME), "culícula", pared celular (PC), núcleo (N), nucleolo $(\mathrm{nu})$, cloroplasto $(\mathrm{C})$, mitocondria (MI), campo de puntuación $(\mathrm{CP})$ y plasmodesmos $(\mathrm{P})$.

Figs. 1-6. Observaciones al iV.O. (1 y 4) y al M.E.T. $(2,3,5$ y 6$)$ de cortes transversales de esporofitos jóvenes de $M$. pyrifera. Figs. 1-3. De dos semanas de edad. Figs. 4-6. De tres semanas de edad. Fig. 1. Talo uniestratificado. Barra $=2 \mathrm{~nm}$. Fig. 2. Célula con una gruesa "cuticula", cloroplasto con 7 bandas de tilacoides y núcleo prominente. Barra =0,2 nm. Fig. 3. Campo de puntuación. Barra $=0,05 \mathrm{~nm}$. Fig. 4. Talo de tres hileras de células. Barra $=2 \mathrm{~m}$. Fig. 5. Campos de puntuación en la pared celular en vista longitudinal. Barra $=0,5 \mathrm{~nm}$. .ig. 6. Campos de puntuación en vista tangencial. Barra $=0,4 \mathrm{~nm}$. 

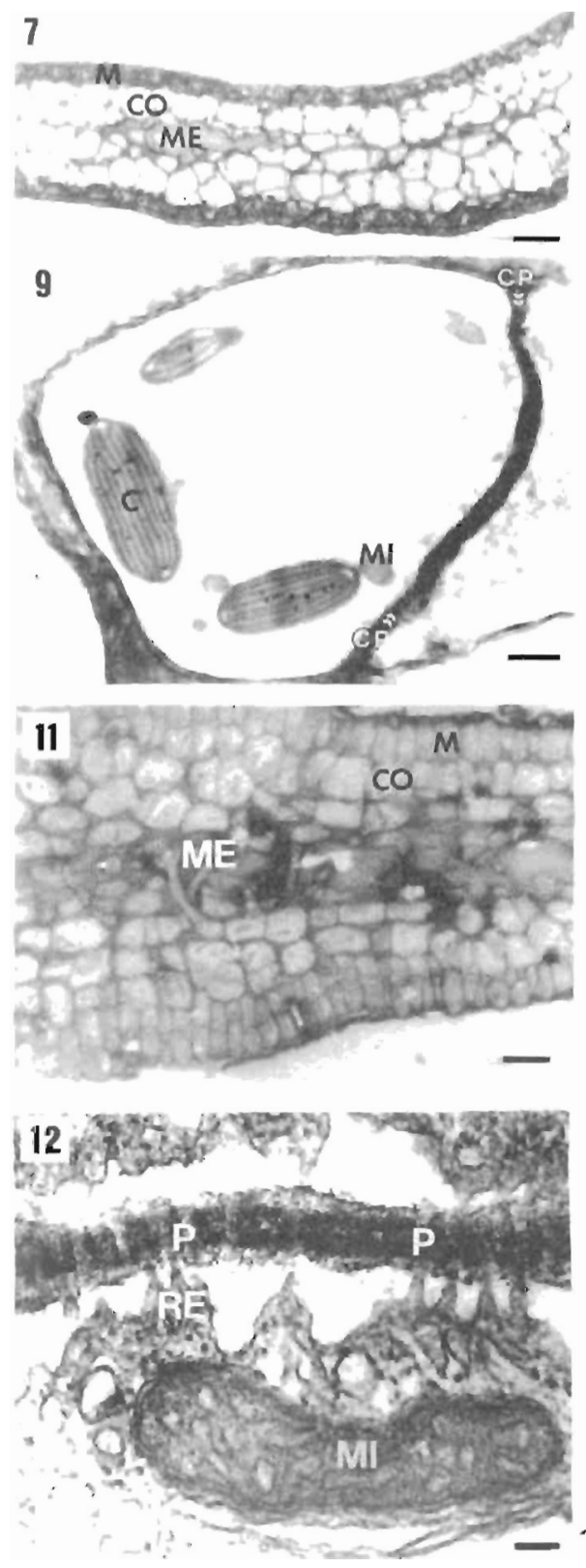
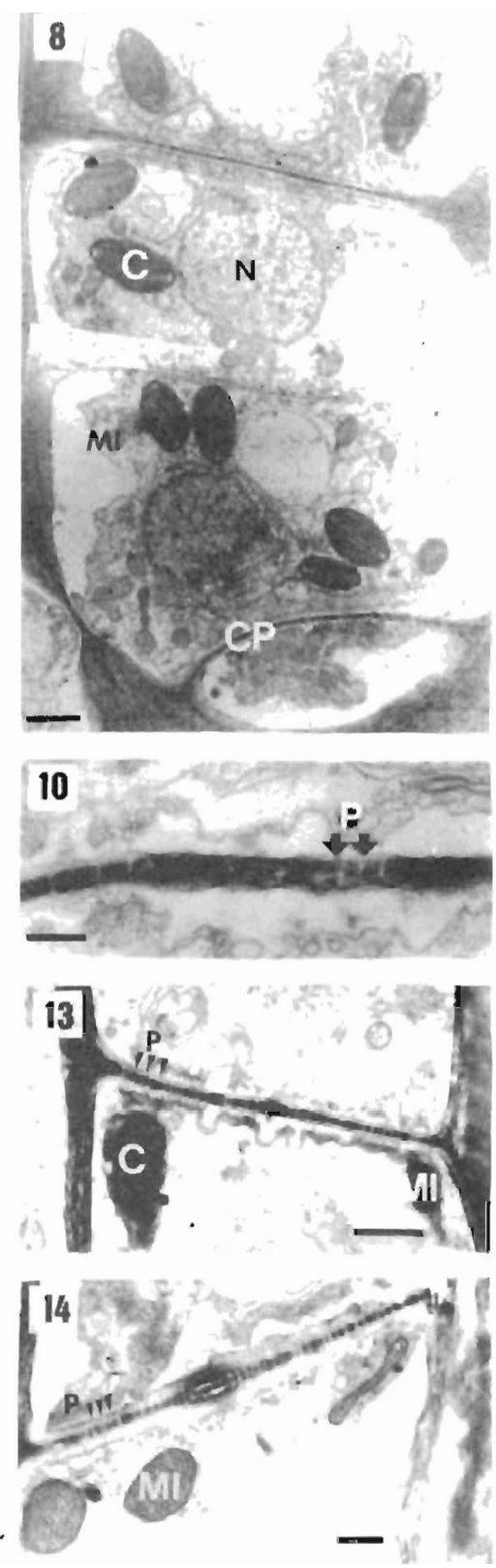

Meristodermo (M), corteza (Co), médula (ME), "cuticula", pared celular (PC), núcleo (N), nucleolo (nu), cloroplasto (C), mitocondria (MI), campo de puntuación (CP) y plasmodesmos (P).

F:gs. 7-14. Observaciones al M. O. (7 y 11) y al M.E.T. (8-10 y 12-14) de cortes transversales de esporofitos jóvenes de $M$. pyrifera. Figs. 7-9. De cinco a seis semanas de edad. Figs. 11-14. De siete a nueve semanas de edad. Fig. 7. Corte longitudinal del talo mostrando meristodermo, corteza y médula. Barra $=20 \mathrm{~nm}$. Fig. 8. Filamento criboso de la médula mostrando un campo de puntuación lateral (flecha) compartido con un filamento adyacente. Barra =1,25 nm. Fig. 9. Elemento criboso con campos de puntuación. Barra $=2,5 \mathrm{~nm}$. Fig. 10. Campos de puntuación con plasmodesmos asociados con sistemas de membranas (flechas). Barra $=0,5 \mathrm{~nm}$. Fig. 11. Corte longitudinal del talo mostrando meristodermo, corteza y médula. Barra $=20 \mathrm{~nm}$. Fig. 12. Area cribosa con plasmodesmos. Barra $=0,5 \mathrm{~nm}$. Figs. 13 y 14. Areas cribosas con plasmodesmos, aparentemente distribuidos al azar, asociados a retículo endoplásmico y mitocondrias. Barra =0,2 nm. 
plántulas incrementan el número de capa., celulare:. Las células del meristodermo son pequeñas y presentan varios cloroplastos similarus a los descritos. Las células corticales son de mayor tamaño, con un núcleo relativamente más pequeño que el de las células del meristodermo, un menor número de cloroplastos y un ciloplasma reducido por la presencia de una gran vacuola central. Se reconocen numerosos campos de puntuación primarios con plasmodesmos. En la región medular, se observan filamentos longitudinales de células de 5 a $10 \mu \mathrm{m}$ de diámetro, con una malriz intercelular amorfa abundante (Figs. 7, 8 y 9). Presentan núcleo, mitocondrias, cloroplastos y un mayor número de vacuolas de menor tamaño que en las células corticales. Las mitocondrias están concentradas en la cercanía de la pared transverial de los filamentos (Fig, 8). Dicha pared, que oscila entre 5 y $10 \mu \mathrm{m}$ de diámctro, se encuentra atravesada por numerosos plasmodesmos con un diámetro de poro de 43,5 $\mathrm{nm}$.

En algunos casos, estos plasmodesmos se encuentran distribuidos at azar; en otros, agrupados en la periferia y relativamente más aislados entre sí en la zona central. En las cercanías de los mismos se puede observar retículo endoplásmico (Figs. 8, 10 y 12). En ocasiones, se pueden ver campos de puntuación primarios con plasmodesmos conectando filamentos medulares contiguos a través de sus paredes laterales (Fig. 8).

Entre la sétima y novena semana de edad, los talos presentan un mayor número de ustratos celulares (Fig. 11) y están constituidos por una lámina, un estípite y rizoides. Fręcuentemente, y tal como en estadios más jóvenes, se observan campos de puntuación primarios comunicando células del meristodermo, células de la corteza y células meristodérmicas y corticales entre sí. En la médula, los filamentos longitudinales presentan plasmodesmos apirentemente dispuesios al azar en sus paredes transversales (Fig. 14), en la cual s observan dos capas. Ia interna más electrodensa que la externa (Fig. 13). Los po- ros presentan un diámetro promedio de 38,5 nım. Además. en la médula existen otros filamentos más delgados, que se disponen oblicua o transversalmente, conectándose directamente con células de la conteza intema (Fig. II).

\section{DISCUSION}

Los esporofitos de Macrocystis prriferade hasta dos semanas de edad presentan un talo uniestratificado con células indiferenciadas. con alta capacidad de división y de realizar fotosíntesis, características que sólo se manlienen en Jas células de los estratos exticnos hasta las nueve semanas de cluración del estudio. Estas características corresponden a las células del meristodermo ya descritas por Fritsch (1945) yarker ( 1971 ) en plantas adultas del orden I aminariales. Las células parenquimáticas de la corteza que se diferencian posteriormente, coincide con lo señalado para esporofitos adultos de $M$. pyifira (Paiker, 1971) y de M. integrifolia (Shih et al., 1983).

La presencia de campos de puntuación con plasmodesmos en el meristodermo y corteza ha sido también señalada para ejemplares adultos de otras especies de algas pardas, además de $M$. plrifera (Parker y Huber, 1965). Así, Davies el al., (1973), Sideman y Scheirer (1977). Schmitz y Kühn (1982) y Chung et al., (1987) Jos citaron en Laminaria, Scrosati (1993) en Lessonia. Bisalputra (1966) en Egregia y Fucus, McCully (1965; 1968) en Fucus y Scrosati (1992) en Desmarestia. Ei diámetro promedio de los poros, aquí presentado, se encuentra comprendido entre los valores dados por dichos autores, aproximadamente entre 30 y $60 \mathrm{~nm}$. En esporofitos jóvenes de $M$. purifera de solo dos células. Gherardini y North (1971) también observaron poros en la pared transversal, pero dispuestos de manera aislada. Las células de la corteza se diferencian posteriomente como resultado de divisiones anticlinales y periclinales, coincidiendo con lo señaludo para esporofitos adultos de $M$. prifera (Parker. 
1971) y de M. integrifolia Bory (Shih et al., 1983).

Los primeros filamentos longitudinales aparecen en la médula a la quinta semana de edad, y crel resultado de la diferenciación de las células más internas de la corteza. Así, paulacinaminte se produce un alargamiento longitudinal de dichas células, formación de nuevos tabiques transversales y producción de abundante matriz intercelular, lo que va sepaando a los filamentos medulares de las células de la corteza interna. Los cambios observados en el protoplasto coinciden, en general, con las observaciones efectuadas sobre procesos semejantes en Nerenclistis (Schmitz \& Srivastavil. 1976), Laminaria (Schmitz \& Srivastava, 1974; Sideman \& Scheirer, 1977) y Alaria (Schmitz \& Srivastava, 1975). Además de los plasmodesmos de la pared transversal de estos filamentos, también se observan campos clc puntuación primarios conectando paredes laterales, estructuras que todavia no han sido descritas hasta el presente en estudios anatómicos de otras especies del orden Laminariales. Es probable que dichas esuructuras se produzcan allí durante la formación de las células, a partir de la corteza interna. Sykes (1908) afirma que en etapas posteriores, la continua separación que se produce entre los filamentos medulares podría ser el principio de conexiones filamentosas entre los mismos a partir de esos campos de puntuación.

A las nueve semanas de cultivo, la médula se va engrosando como resultado de la incorporación de nuevos filamentos longitudinales que se diferencian desde la corteza, de la conrinua formación de matriz intercelular y de la producción de los filamentos Ilamados «hifas» (Sykes. 1908; Fritsch, 1945) o filamentos asociados (Parker \& Huber, 1965) por parte de las células de la cortezá interna.

Es ya conocida la importancia que tienen los «tubos cribosos»' o filamentos cribosos perimeciulares en la translocación de sustancias a lo largo del talo adulto de Macrocysis (Parker, 1965: 1966; Shih et al., 1983). Las células que los componen (elementos cribosos) tienen placas cribosas con poros encre $0.45 \mu \mathrm{m}$ y $4.50 \mu \mathrm{m}$ de diámetro, según las especies, la edad de la planta y la ubicación anatómica (Parker \& Huber, 1965 ; Schmitz, 1981; Hay, 1986; Núñez \& Alveal, 1988). También se señala la presencia de otros filamentos en la médula, de posición más interna, los llamados filamentos trompeta (Smith, 1939) o filamentos cribosos medulares (Esau, 1969). Los elementos cribosos que lo constituyen poseen áreas cribosas (término utilizado aquí según Esau, 1969; Sideman \& Scheirer, 1977; Schmitz, 1990), con poros de mucho menor diámetro (Parker \& Huber, 1965). Los filamentos trompera serían homólogos a los «tubos cribosos», pero se formarían en etapas más tempranas de la vida del esporofito. La característica forma de trompeta se produce debido a la tensión diferencial al crecer la planta en longitud; además, los poros finalmente se ocluyen con calosa, perdiendo así su capacidad de translocación (Sykes, 1908; Schmitz, 1990) En el caso de los filamentos medulares longitudinales aquí descritos, su ubicación y las características de sus paredes transversales y de los poros que hay en ella purmiten suponer que se trata de lo: primeros filarıenros cribosos en formación, los que se diferenciarian en filamentos trompeta al crecer la planta. Los grupos de poros hallados en la periferia de las paredes transversales de algunos filamentos longitudinales medulares han sido observados en ápices de plantas adultas. Sykes (1908) postula que esas estructuras serían etapas intermedias en la formación de los grandes poros de los "tubus cribosos", en un proceso que involucra la síntesis y posterior degradación de materiales de la pared celular, dado que el diámetro de los poros encontra-

'El término «lubos cribosos» figura aqui entre comillas porque, si bien eslas estruclulas de las Laminariales son bastante parecidas a los lubos cribosos del floema de las plantas vasculares terrestres. difieren en algunos aspecios impoltantes (Esau, 1969: Schmilz. 1990) 
dos en las paredes transversales de estos primeros filamentos cribosos es semejante al de los campos de puntuación primarios y, debido a que no hay diferencias ultraestructurales importantes entre ambos, se ha llamado plasmodesmos a las conexiones citoplasmáticas que los atraviesan, criterio seguido también por Parker \& Huber (1965) y Parker (1971). Dichas paredes transversales podrían ser consideradas como campos de puntuación primarios especializados (Sideman \& Scheirer, 1977). El diámetro de poro medido aquí en estas paredes es uno de los menores entre los encontrados en elementos cribosos de ejemplares macroscópicos de otras algas pardas (Schmitz, 1990). Para determinar si los filamentos medulares longitudinales de talos de 9 semanas de $M$. prrifera cumplen ya la función de translocación de fotosintatos, es necesario lleval a cabo estudios fisiológicos.

En este estudio se han reconocido aspectos de la ontogenia de las erapas juveniles de los esporofitos de $M$. pyrifera.

Agradecimientos: Se agradecen las sugerencias y críticas recibidas de Krisler Alveal, Héctor Romo y Ricardo Scrosati. César Córdova agradece la beca otorgada por la Red Latinoamericana de Botánica que permitió realizar el presente trabajo en la Universidad de Concepción, Chile. Del mismo modo, se agradece el apoyo recibido del personal del Laboralorio de Microscopía Electrónica y del Laboratorio de Cultivo de Algas de la misma Universidad por su valiosa colaboración para el desarrollo del presente trabajo.

\section{BIBLIOGRAFIA}

BARRAI,ES. H. L.. PETERSON, R. L., GRFNVILLE, D. J. \& GERRATH, F., 1981. Use of clearing and fluorescence techniques in anatomical studies of the sporophytc of Macrocrsis (Phaeophyceae. Laminarialcs). Phycologia 20(4): 392-396.

BISALPUTRA. T. 1966. Electron microscopy study of the protoplasmic continuily in certain brown algae. Can, J. Bot. 44: 8993.

CHUNG. 1. K.. LEDBETTER, M. C. \&
BRINKHUIS, B.H. 1987. Finc structure of Laminaria saccharina (L.) Lamour. The Korean J. Phycol. 2(2): |47-17|

DAVIES. J. M., FERRIER, N. C. \& JOHNSTON. C. S. 1973. The ultrastructure of the meristoderm cells of the hapteron of Laminaria. J. Mar. Biol. Ass. U. K. 53: 237 246.

ESAU, K. 1969. The phloem. In: LINSBAUER. K. (Ed.). Handbuch der Ptlanzenanatomie. 5(2). Gebruder Borntraeger, Berlin. pp. 263-27!

FRITSCH: F. E. 1945. The structure and reproduction of algac. Vol. 2. London: Cambridge University Press, 939 pp.

GHERARDINI. G. L. \& NORTH. W. J. 1971. Electron microscopic sludies of Macrocystis pyrifera zoospore.. gametophytes and early sporophytes. Proc. Seventh Int Seaweed Symp. 172-180.

GLAUERT, A. U. 1974. Fixation, dehydration and embedding of biological spcuimens. In GLAUERT, A. U. (Ed.) Practical methods: in electron microscopy. Amsterdam. North Holland Publishing Co., pp. I-20l .

GRENVILLE, D. J.; PETHRSON, R. L.; BARRALES, H. L. \& UERRATH, J. F. 1982. Structure and development of the secretory cells and ducts system in Macrocystis pyrifera (L.) C. Agardh. J. Phycol. 18: 232240.

HAY, C. H. 1986. A new spccies of Macrocistis C. Ag. (Phaeophyla) from Marion Island, southern Indian ocean. Phycologia 25(2): 24!-252.

KÜHNEMANN, O. 1970. Algunas consichraciones sobre los bosques de Macrocystis pyrifera. Physis 29(79): 273-296.

- 1972. Bosquejo filogeográfico de la vegrelación marina del litoral argentino. Physis 31(82): $117-142$.

LOBBAN, C. S. 1978. The growth and death of the Macrocystis sporophyte (Phaenphyccic. Laminariales). Phycologia 17(2): 196-212.

McCulLY. M. E. 1965. A note on the structures of the cell walls of the brown alga Fucus. Can. J. Bot. 43: 1001-1004.

McCULLY, M. E. 1968. Histological studies on the genus Fucus III. Fine structure and possible functions of the cpidermal cells of the vegetative thallus. J. Cell. Sci, 3: 1-16.

NUNEEZ. O.M. \& ALVEAL, K. 1988. Estructuras 
cribadas en algunas algas pardas chilenas Gayana Bol. 45(1-4): 371-378.

OLIVER. F. W. 1887 . On the obliteration of the sieve lubes in Laminarieae. Ann. Bot. I(2): 95-117.

PARKER, B. C. 1965. Translocation in the giant kelp Macrocrstis I. Rales, direction quanticy of $\mathrm{C}^{\text {st }}$-labeled products and fluoreccin. J. Phycol 1: 41-46

PARKER. B. C. 1966. Translocalion in Macrocistis III. Composition of sieve tube exudatc and identification of the major $\mathrm{C}^{1+}$ labelcd products. J. Phycol. 2:38-41.

PARKI:R, B. C. 1971. The internal structure of Macrocystis. In: NORTH, W. J. (Ed.) The Biology of Giant Kclp Beds (Macrocistis) in California. Beinefte zur Nova Hedwigia 32: $99-121$

PARKER. B. C. \& HUBER. J. 1965. Translocation in Macrocisris II. Fine structure of the sieve tubes. J. Phycol. 1: 172-179.

PARKER, B. C. \& DIBOLL, A. G. 1966. Alcian stains for histoche mical localization of acid and sulphate polysaccharides in algae. Phycologia 6: 37-46.

RAMIREZ. M. E. \& SA.VTELICES, B. 1991 Curálogo de las algas marinas bentónicas de la cusla iemplada del Pacífico de Sudamérica. Sanciago, Publicaciones Periódicas Pontificia Universidad Cacólica de Chilc. Monogr. Biol. 5: 1-437

REYNOLDS. E. S. 1963. The use of lead citrate at high $\mathrm{pH}$ as an electron opaque stain in electron microscopy. J. Cell. Biol. 17: 208$2 \mid 2$.

RICKER. R. W. 1987. Taxonomy and biogeography of Mcquarie Island Seaweeds. Dorchester, Henry Ling Lid. The Dorset Press, $344 \mathrm{p}$

SCHMITZ, K. 1981. Translocation. In: LOBBAN, C. S. \& WYNNE. M. J. (Eds.) The Biology of Seaweeds. Botanical Monographs. 17. OxFord. Blackwell Sci. Publ.. pp. 534-558. SCHMITZ. K. 1990. Algac. In: BEHNKE, H. D
\& SJOLUND. R. D. (Lids.) Sieve Elements Berlin, Springer - Verlag. pp. 1 - 18.

SCHMITZ. K. \& SRIVASTAVA, L. M. 1974. Fine structurc and development of sieve tubes in Laminaria groenlandica Rosenv Cycobiologie 10(1): 66-87

SCHMITZ, K. \& SRIVASTAVA, L. M. 1975. On the fine structure of sieve tubes and the physiology of assimilatc transport in Alaria marginata Postels el Ruprecht. Can. I. Bot. 53(9): 861876

SCHMITZ, K. \& SRIVASTAVA. L. M. 1976. The fine struclure of sieve elements of Nereocistis lutkeana Anı. J. Bol. 63 (5): 079-693

SCHMITZ. K. \& KÜHN. R. 1982. Fine structure, distribution and frequency of plasmodesmata and pits in the cortex of Laminaria hrperborea and $L$ saccharina. Planla 154: 385-392

SCROSATI. R. A. 1992. Estudio anatómico de un morlolipo ligulado de Desmarestia (Phacophyceae. Desmarestiales) de Chile central. Gayana Bot. 49 (1-4): 17-24.

SCROSATL. R. A. 1993. Estudio anatónico de Lessonia nigrescens (Phacophyceac Laminariales) de Chile. Darwiniana 32(14): $27 !-278$

SHJH, M. L., FLOCH, J. Y. \& SRIVASTAVA, L. M. 1983. Localization of $C^{14}$-labeled assimilates in sicve elements of Macrocrstis integrifolia by histoautoradiography. $C_{i t n}$ J. Bol. 61: 157-163.

SIDEMAV, E. J. \& SCHEIRER, D C. 1977. Sume fine slructural observations on developing and mature sieve elements in the brown alga Lamimaria saceharina. Am. J. Bot. 67(0): 649-657.

SMITH. A. I. 1939. The comparative histology ol some of the Laminariales. Am. J. Bot. 26 $571-585$.

SYKES. M. G. 1908. Analomy and histology of Macrocystis prifera and Laminaria saccharina. Ann. Bor. 22(86): $291-325$ 